\title{
Identification of key genes and biological processes contributing to colitis associated dysplasia in ulcerative colitis
}

\author{
Di Zhang ${ }^{1,2}$, Pengguang Yan ${ }^{1,2}$, Taotao Han ${ }^{1,2}$, Xiaoyun Cheng ${ }^{1,2}$, Jingnan $\mathbf{L i}^{\text {Corresp. 1,2 }}$ \\ ${ }^{1}$ Department of Gastroenterology, Peking Union Medical College Hospital, Peking Union Medical College, Chinese Academy of Medical Sciences, Beijing, \\ China \\ 2 Key Laboratory of Gut Microbiota Translational Medicine Research, Chinese Academy of Medical Sciences, Beijing, China \\ Corresponding Author: Jingnan Li \\ Email address: pumcjnl@126.com
}

Background. Ulcerative colitis-associated colorectal cancer (UC-CRC) is a life-threatening complication of ulcerative colitis (UC). The mechanisms underlying UC-CRC remain to be elucidated. The purpose of this study was to explore the key genes and biological processes contributing to colitis-associated dysplasia (CAD) or carcinogenesis in UC via database mining, thus offering opportunities for early prediction and intervention of UC-CRC.

Methods. Microarray datasets (GSE47908 and GSE87466) were downloaded from Gene Expression Omnibus (GEO). Differentially expressed genes (DEGs) between groups of GSE47908 were identified using the "limma" R package. Weighted gene co-expression network analysis (WGCNA) based on DEGs between the CAD and control groups was conducted subsequently. Functional enrichment analysis was performed, and hub genes of selected modules were identified using the "clusterProfiler" R package. Single-gene gene set enrichment analysis (GSEA) was conducted to predict significant biological processes and pathways associated with the specified gene.

Results. Six functional modules were identified based on 4929 DEGs. Green and blue modules were selected because of their consistent correlation with UC and CAD, and the highest correlation coefficient with the progress of UC-associated carcinogenesis. Functional enrichment analysis revealed that genes of these two modules were significantly enriched in biological processes, including mitochondrial dysfunction, cell-cell junction, and immune responses. However, GSEA based on differential expression analysis between sporadic colorectal cancer (CRC) and normal controls from The Cancer Genome Atlas (TCGA) indicated that mitochondrial dysfunction may not be the major carcinogenic mechanism underlying sporadic CRC. Thirteen hub genes (SLC25A3, ACO2, AIFM1, ATP5A1, DLD, TFE3, UQCRC1, ADIPOR2, SLC35D1, TOR1AIP1, PRR5L, ATOX1, and DTX3) were identified. Their expression trends were validated in UC patients of GSE87466, and their potential carcinogenic effects in UC were supported by their known functions and other relevant studies reported in the literature. Single-gene GSEA indicated that biological processes and Kyoto Encyclopedia of Genes and Genomes (KEGG) pathways related to angiogenesis and immune response were positively correlated with the upregulation of TFE3, whereas those related to mitochondrial function and energy metabolism were negatively correlated with the upregulation of TFE3.

Conclusions. Using WGCNA, this study found two gene modules that were significantly correlated with $C A D$, of which 13 hub genes were identified as the potential key genes. The critical biological processes in which the genes of these two modules were significantly enriched include mitochondrial dysfunction, cell-cell junction, and immune responses. TFE3, a transcription factor related to mitochondrial function and cancers, may play a central role in UC-associated carcinogenesis.

Peer] reviewing PDF | (2020:12:56299:2:0:NEW 25 Mar 2021) 


\section{Identification of key genes and biological processes}

2 contributing to colitis associated dysplasia in

3 ulcerative colitis

4

5

Di Zhang ${ }^{1,2}$, Pengguang Yan ${ }^{1,2}$, Taotao Han ${ }^{1,2}$, Xiaoyun Cheng ${ }^{1,2}$, Jingnan $\operatorname{Li}^{1,2}$

$7 \quad{ }^{1}$ Department of Gastroenterology, Peking Union Medical College Hospital, Peking Union

8 Medical College, Chinese Academy of Medical Sciences, Beijing, China

$9{ }^{2}$ Key Laboratory of Gut Microbiota Translational Medicine Research, Chinese Academy of

10 Medical Sciences, Beijing, China

11

12 Corresponding Author:

13 Jingnan $\mathrm{Li}^{1,2}$

14 No.1 Shuaifuyuan Dongcheng District, Beijing, 100730, China

15 Email address: pumcjnl@126.com 
18

19

20

21

22

23

24

25

26

27

28

29

30

31

32

33

34

35

36

37

38

39

40

41

42

43

44

45

46

47

48

49

50

51

52

53

54

55

56

57

\section{Abstract}

Background. Ulcerative colitis-associated colorectal cancer (UC-CRC) is a life-threatening complication of ulcerative colitis (UC). The mechanisms underlying UC-CRC remain to be elucidated. The purpose of this study was to explore the key genes and biological processes contributing to colitis-associated dysplasia (CAD) or carcinogenesis in UC via database mining, thus offering opportunities for early prediction and intervention of UC-CRC.

Methods. Microarray datasets (GSE47908 and GSE87466) were downloaded from Gene Expression Omnibus (GEO). Differentially expressed genes (DEGs) between groups of GSE47908 were identified using the "limma" R package. Weighted gene co-expression network analysis (WGCNA) based on DEGs between the CAD and control groups was conducted subsequently. Functional enrichment analysis was performed, and hub genes of selected modules were identified using the "clusterProfiler" R package. Single-gene gene set enrichment analysis (GSEA) was conducted to predict significant biological processes and pathways associated with the specified gene.

Results. Six functional modules were identified based on 4929 DEGs. Green and blue modules were selected because of their consistent correlation with UC and CAD, and the highest correlation coefficient with the progress of UC-associated carcinogenesis. Functional enrichment analysis revealed that genes of these two modules were significantly enriched in biological processes, including mitochondrial dysfunction, cell-cell junction, and immune responses. However, GSEA based on differential expression analysis between sporadic colorectal cancer (CRC) and normal controls from The Cancer Genome Atlas (TCGA) indicated that mitochondrial dysfunction may not be the major carcinogenic mechanism underlying sporadic CRC. Thirteen hub genes (SLC25A3, ACO2, AIFM1, ATP5A1, DLD, TFE3, UQCRC1, ADIPOR 2, SLC35D1, TOR1AIP1, PRR5L, ATOX1, and DTX3) were identified. Their expression trends were validated in UC patients of GSE87466, and their potential carcinogenic effects in UC were supported by their known functions and other relevant studies reported in the literature. Single-gene GSEA indicated that biological processes and Kyoto Encyclopedia of Genes and Genomes (KEGG) pathways related to angiogenesis and immune response were positively correlated with the upregulation of TFE3, whereas those related to mitochondrial function and energy metabolism were negatively correlated with the upregulation of TFE3.

Conclusions. Using WGCNA, this study found two gene modules that were significantly correlated with CAD, of which 13 hub genes were identified as the potential key genes. The critical biological processes in which the genes of these two modules were significantly enriched include mitochondrial dysfunction, cell-cell junction, and immune responses. TFE3, a transcription factor related to mitochondrial function and cancers, may play a central role in UCassociated carcinogenesis.

\section{Introduction}

Ulcerative colitis (UC) is a subtype of inflammatory bowel disease (IBD), which is characterized by long-standing and recurring chronic inflammation of the colonic mucosa. UC-associated 
58

59

60

61

62

63

64

65

66

67

68

69

70

71

72

73

74

75

76

77

78

79

80

81

82

83

84

85

86

87

88

89

90

91

92

93

94

95

96

97

colorectal cancer (UC-CRC) is the most severe and life-threatening complication, especially in patients suffering from UC for a long duration. Epidemiological data have revealed that the risk of UC-CRC increases with disease duration (Bernstein et al. 2001; Bopanna et al. 2017).

Although more attention has been given to the colonoscopic screening of dysplasia and cancer in UC patients(Laine et al. 2015; Rubin et al. 2019), early diagnosis is difficult because of the flat appearance and multifocal lesions. Therefore, there is an urgent need to explore the molecular biological mechanisms underlying UC-associated carcinogenesis and to find new strategies for early diagnosis, treatment, and prevention of UC-CRC.

Early genetic changes in precancerous lesions have been reported to contribute to the initiation of carcinogenesis. For example, Ju et al. revealed that $70 \%$ of miRNA alterations occur during the transition from normal to a preneoplastic stage of breast cancer(Ju et al. 2020). Similarly, Zhang et al. found that most of the differentially expressed genes (DEGs) identified in highgrade intraepithelial neoplasia (HGIN) and early gastric cancer (EGC) compared to their paired controls have already changed in low-grade intraepithelial neoplasia (LGIN) lesions. In addition, they identified 22 coDEGs (co-up DEGs and co-down DEGs), which are thought to play crucial roles in gastric tumorigenesis and progression, during the stages of LGIN, HGIN, EGC, and gastric adenocarcinoma, (Zhang et al. 2020). This phenomenon offers the possibility of early diagnosis and treatment of cancers in patients with UC. In fact, some abnormal molecular events have also been demonstrated in the inflamed colonic mucosa of UC before any apparent histological evidence of colitis-associated dysplasia (CAD) or cancer(Itzkowitz 2006; Itzkowitz \& Yio 2004; Tang et al. 2012). However, comprehensive analysis based on gene expression profiles to reveal the genetic changes that occur in UC and contribute to carcinogenesis is still lacking.

In the present study, using database mining, we explored the key genes and biological processes contributing to CAD that were dysregulated in UC. We conducted weighted gene coexpression network analysis (WGCNA) based on DEGs between CAD and control groups and found two gene modules correlated with the progression of UC-associated carcinogenesis. Functional enrichment analysis of the genes in these two modules revealed the associated crucial biological processes. Thirteen hub genes were identified as the potential key genes. Furthermore, we investigated the changes in their expression in the UC and CAD groups.

\section{Materials \& Methods}

\section{Data selection and processing}

By searching the Gene Expression Omnibus (GEO, https://www.ncbi.nlm.nih.gov/geo/) database, the microarray dataset GSE47908 based on the GPL570 platform was selected for analysis because it embraces the transcriptional profiles of colonic mucosa from both UC and CAD patients(Bjerrum et al. 2014). Specifically, it included data from 45 patients with UC (20 with left-sided colitis, 19 with pancolitis, and six with UC-associated dysplasia) and 15 healthy controls. After obtaining the data, principal component analysis (PCA) was performed to check and visualize the grouped data. For validation, another microarray dataset GSE87466 comprising 
the gene expression profiles of colonic mucosa from 87 patients with UC and 21 healthy controls was selected to check the gene expression trends in UC(Li et al. 2018). For comparison, we downloaded the transcriptome and clinical data of 480 patients with sporadic colorectal cancer (CRC) and 41 healthy controls from The Cancer Genome Atlas (TCGA) database (date limit: February 23, 2021), using "TCGAbiolinks" R package version 2.16.4. The gene expression profiles were normalized using the voom function in "limma" $R$ package(Ritchie et al. 2015).

\section{Differential expression analysis}

Differential expression analysis was performed using the "limma" $\mathrm{R}$ package(Ritchie et al. 2015). The sum of the mean value of absolute $\log _{2}$ fold change (FC) and the two standard deviations of absolute $\log _{2} \mathrm{FC}$ was used as the cut-off value of $\log _{2} \mathrm{FC}$. Genes with absolute $\log _{2}$ $\mathrm{FC}>\log _{2} \mathrm{FC}$ cut-off and adjusted $\mathrm{p}$ value $<0.05$, were considered as DEGs. The results are visualized as volcano plots.

\section{WGCNA}

The expression profiles of DEGs between CAD and control group were extracted to construct weighted gene co-expression network using R package "WGCNA"(Langfelder \& Horvath 2008; Zhang \& Horvath 2005). First, the candidate soft-thresholding powers (1 to 30) were used to calculate the scale independence and mean connectivity using the pickSoftThreshold function. The first candidate power, whose degree of independence was $>0.8$, was selected as the proper power. Then, a co-expression network was constructed and modules were identified using the blockwiseModules function, with the parameters mergeCutHeight set to 0.28 and minModuleSize set to 30. Each module was assigned a unique color. Finally, the Pearson correlation coefficient and corresponding $p$ value between each module's eigengene and phenotype were calculated. In addition to the UC and CAD phenotypes, another phenotype "Progress" was also included to quantify the dynamic process of UC-associated carcinogenesis. The phenotype "Progress" was assigned the values of 0,1 , and 2 , to represent the control, UC, and CAD groups, respectively; this reflected the progressive process from normal to UC and then to CAD. It means that if a module is significantly correlated to "Progress", then this module is considered to be correlated to the progressive process from normal to CAD.

\section{Identification of hub genes}

For a specified module gene, the module membership (MM) calculated by signedKME function represents its degree of importance in the module, and gene significance (GS) calculated by the cor function represents the degree of correlation with the phenotype. By calculating MM and GS, the module genes with high MM and GS can be defined as hub genes. In the present study, thresholds of MM $>0.9$ and GS $>0.7$, were chosen to screen for the hub genes of each module(Zou et al. 2019). 


\section{Function enrichment analysis}

138 Functional enrichment analysis was performed using the "clusterProfiler" R package(Yu et al.

139 2012). Significantly enriched Gene Ontology-Biological processes (GO-BP) and Kyoto

140 Encyclopedia of Genes and Genomes (KEGG) pathways were identified using enrichGO and

141 enrichKEGG functions, respectively. Gene set enrichment analysis (GSEA) was performed

142 based on the gene list sorted by $\log _{2} \mathrm{FC}$ obtained from differential expression analysis using

143 gseGO and gseKEGG functions (Subramanian et al. 2005). Single-gene GSEA was conducted

144 based on the gene list sorted by Spearman correlation coefficient between every gene and the

145 specified hub gene to predict the significant biological processes and pathways associated with

146 the hub gene. A $p$ value $<0.05$ was considered significant.

147

148

\section{Results}

149

\section{GEO data overview}

150

A flow chart of the present study is shown in Fig. 1A. As for GSE47908 dataset, 15 controls, 20

151

152

patients with left-sided colitis, and six patients with UC-associated dysplasia were enrolled in the

153 present study, representing the control, UC, and CAD groups, respectively. Clinical data including sex, age, disease duration, Mayo score, Mayo endoscopic score, smoking status, and daily medication were extracted and compared using the chi-squared test(Bjerrum et al. 2014). Results showed that there were no significant differences in sex, disease duration, smoking status, or daily medication between the groups. As for age, Mayo score, and Mayo endoscopic score, only median and interquartile ranges (IQR) were known, and the corresponding p-values could not be calculated (Table S1). After processing, four outliers were removed and a total of 37 samples, including 13 controls, 18 patients with UC, and six patients with CAD, were enrolled. As shown in the PCA plot (Fig. 1B), the gene transcriptional profiles of these three groups were clearly distinct from each other. The PCA plot of gene expression profiles of 87 patients with UC and 21 healthy controls in GSE87466 dataset shown in Fig. 1C, also indicates a significant distinction between these two groups.

164

\section{Differential expression analysis}

166

To investigate the genetic changes during the progress from normal to $\mathrm{UC}$ and then to CAD, differential expression analyses between UC and control, CAD and UC, and CAD and control groups in GSE47908 were performed. The results are presented in Fig. 2. There were 679 upregulated and 288 downregulated genes in UC compared to those in the control group (Fig. $2 \mathrm{~A}, \log _{2} \mathrm{FC}$ cut-off of 1.092), 260 upregulated and 616 downregulated genes in CAD compared to those in the UC group (Fig. 2B, $\log _{2}$ FC cut-off of 1.073), and 305 upregulated and 462 downregulated genes in CAD compared to those in the control group (Fig. $2 \mathrm{C}, \log _{2} \mathrm{FC}$ cut-off of 0.804). Venn diagrams were then used to further detail the genetic changes during this dynamic process. There were 67 co-up DEGs (Fig. 2D) and 46 co-down DEGs (Fig. 2E) in UC vs. controls and CAD vs. controls. In addition, the Venn diagram shows that among 260 upregulated 
177 downregulated genes (Fig. 2E) changed significantly compared to the control group. This

178 indicates that some DEGs dysregulated during the progress from normal to UC may continue to

179 change in $\mathrm{CAD}$ and even contribute to the initiation of $\mathrm{CAD}$, while others might be only

180 responsible for the development of UC and may change back to the normal level if the

181 inflammation is controlled.

182 To gain further insights into the expression pattern of DEGs during the progress from normal

183 to UC and then to CAD, the top 40 upregulated and top 40 downregulated DEGs between CAD

184 and controls after sorting by adjusted p-value were visualized in a heat map (Fig. 2F). Row

185 cluster analysis showed that these DEGs were grouped under two clusters: upregulated and

186 downregulated genes in CAD compared to those in controls. However, when UC samples were

187 taken into consideration, every cluster could be further divided into two sub-clusters, showing

188 different changing trends of these DEGs during the progress from normal to CAD.

189 It should be noted that no co-up or co-down DEGs in UC vs. control groups and CAD vs. UC

190 groups were identified in Venn diagrams, while the heatmap showed marked continuous changes

191 in the expression patterns of some DEGs from control to UC and then to CAD. The traditional

192 filter method for DEGs can potentially miss somekey information. To make our analysis more

193 comprehensive, the cut-off criteria of $\log _{2} \mathrm{FC}$ was set to 0.3 , leading to the identification of 4929

194 DEGs between the CAD and control groups, which is a suitable number of DEGs for WGCNA

195 analysis.

196

197

\section{WGCNA}

198

199

WGCNA was performed using 4929 DEGs between the CAD and control groups. By setting the soft threshold to 12 (Fig. 3A-B), a total of six functional modules, apart from the gray module,

200 were identified (Fig. 3C). The turquoise, red, and blue modules were positively correlated with

201 $\mathrm{CAD}$, and the brown, yellow, and green modules were negatively correlated with CAD.

202 Furthermore, the green and blue modules were correlated with UC and CAD in the same

203

204 direction respectively, and were found to be correlated with "Progress" with the highest

205 correlation coefficients (green module: correlation coefficient $=-0.75$, $p$-value $=9 \mathrm{e}-08$; blue module: correlation coefficient $=0.71, \mathrm{p}$-value $=9 \mathrm{e}-07)($ Fig. 3D). This indicates that genes

206 belonging to blue and green modules and the relevant biological processes begin to change at an

207 earlier stage and continue to change throughout the whole process of progression from normal to 208 UC and then to CAD.

209

210

\section{Function enrichment analysis}

211 The GO-BP enrichment analysis of each module is shown in Table S2. Green module genes were

212 significantly enriched in biological processes related to mitochondrial function and energy

213 metabolism (Fig. 4A). Consistent with this observation, KEGG pathway enrichment analysis

214 indicated that the green module genes were significantly enriched in energy metabolism-related

215 pathways, such as the TCA cycle, carbon metabolism, and oxidative phosphorylation (Fig. 4B).

216 Blue module genes were significantly enriched in biological processes related to cell-cell 
217 junctions and immune responses, especially neutrophil-mediated immunity (Fig. 4C). The

218 KEGG pathways that blue module genes were significantly enriched in included infection219 related pathways, focal adhesion, tight junction, Hippo signaling pathway, Notch signaling 220 pathway, and ErbB signaling pathway (Fig. 4D).

221 To further explain the role/s of mitochondrial dysfunction during the process of UC-associated 222 carcinogenesis, GSEA was performed to analyze the alterations in mitochondrial function-related 223 GO-BP in the UC and CAD groups. The results indicated that mitochondrial function-related 224 GO-BP, including cellular respiration, oxidative phosphorylation, respiratory electron transport 225 chain, and mitochondrial gene expression, were all significantly downregulated in UC (Fig. S1A) 226 and CAD (Fig. S1B) groups as compared to those observed for the control group.

To explore the differences between UC-CRC and sporadic CRC in terms of the alteration of mitochondrial functions, the gene expression profiles of 480 sporadic CRC patients and 41 healthy controls from the TCGA database were analyzed. After differential expression analysis, GSEA of GO-BP was conducted based on the gene list sorted by $\log _{2} \mathrm{FC}$. The results showed that biological processes, including mitochondrial gene expression and mitochondrial translation, were upregulated in sporadic CRC patients, and no other mitochondrial function-related biological processes were identified (Table S3). This indicates that the carcinogenic mechanism underlying sporadic CRC is different from that of UC-CRC.

\section{Identification and validation of hub genes}

Based on MM $>0.9$ and GS $>0.7$, seven genes (SLC25A3, ACO2, AIFM1, ATP5A1, DLD, TFE3, and $U Q C R C 1$ ) were identified as hub genes in the green module (Fig. 5A) and six hub genes (ADIPOR2, SLC35D1, TOR1AIP1, PRR5L, ATOX1, and DTX3) were identified in the blue module (Fig. 5C). The differential expression patterns of these 13 hub genes during the progression from normal to UC and then to CAD are displayed in Fig. 5B and Fig. 5D. All of them were observed to be continuously up/downregulated significantly, supporting their potential persistent roles in UC-associated carcinogenesis.

Considering the small sample size of the GSE47908 dataset, validation using another dataset was considered indispensable. To validate the changes in the inflammatory phase, differential expression analysis of the 13 hub genes was performed in UC patients from GSE87466 dataset (87 patients with UC and 21 controls). The results showed that the expression changes of hub genes in UC patients of GSE87466 dataset were consistent with those observed for patients belonging to GSE47908 dataset (Fig. 6). To further explore the potential association of hub genes with UC-associated carcinogenesis, a brief literature review was performed to search for the proof of their carcinogenic effects(Bruggemann et al. 2017; Byeon et al. 2010; Chung et al. 2017; Ciccarone et al. 2020; Ding et al. 2020; Jana et al. 2020; Karginova et al. 2019; Khalil 2007; Kim et al. 2019; Laiho et al. 2003; Li et al. 2019; Linehan et al. 2019; Liu et al. 2018; Millan \& Huerta 2009; Oehler et al. 2009; Perera et al. 2015; Rao et al. 2019; Seth et al. 2009; Thedieck et al. 2007; Viola et al. 2017; Wang et al. 2013; Wang et al. 2020). The results are presented in Table 1. With the exception of TORIAIP1 and PRR5L, almost all other hub genes 
257 have been reported to be involved in cancers. Furthermore, five of the hub genes (AIFM1, 258 ATP5A1, UQCRC1, ADIPOR2, and ATOX1) may play roles in the carcinogenesis of sporadic 259 CRC. These known associations between hub genes and cancers suggest the possible

260

261

262

263

264

265

266

267

268

269

270

271

272

273

274

275

276

277

278

279

280

281

282

283

284

285

286

287

288

289

290

291

292

293

294

295

296

carcinogenic effects of these hub genes in CAD.

\section{Predication of the potential function of TFE3 in UC-associated carcinogenesis by GSEA}

Among the proteins encoded by the hub genes, TFE3, a transcription factor, has been known to be involved in the onset and progress of cancers by regulating many biological processes, such as energy metabolism, lysosomal biogenesis, and immune response(Beckmann et al. 1990; Perera et al. 2015; Willett et al. 2017). In this study, we found that TFE3 was continuously upregulated during UC-associated carcinogenesis. However, the plausible roles of TFE3 in this process remain unclear. Single-gene GSEA revealed that biological processes and KEGG pathways related to angiogenesis and immune response were positively correlated with the upregulation of TFE3 (Fig. 7A, 7C), whereas those related to mitochondrial function and energy metabolism were negatively correlated with the TFE3 upregulation(Fig. 7B, 7D).

\section{Discussion}

Most traditional research related to understanding of carcinogenic mechanisms focuses on comparison between tumor and normal tissue; however, this idea seems to be not fully applicable to UC-CRC considering its gradual molecular changes from inflammation to cancer. As UC$\mathrm{CRC}$ arises due to the chronic inflammation of the colonic mucosa, it is logical to find the carcinogenic genes and biological processes associated with inflammation during the progression of UC(Itzkowitz \& Yio 2004). In the present study, we identified two functional modules by WGCNA using the transcriptional profiles of colonic mucosa from healthy individuals and UC and CAD patients, which are correlated with $\mathrm{UC}$ and $\mathrm{CAD}$ in the same direction, suggesting their potential carcinogenic function when they contribute to inflammation. Thirteen hub genes (SLC25A3, ACO2, AIFM1, ATP5A1, DLD, TFE3, UQCRC1, SLC35D1, TOR1AIP1, PRR5L, $A T O X 1$, and $D T X 3)$ were identified, and their continuous up/down-regulation supported their continuous roles during the process of progression from inflammation to carcinogenesis.

Functional enrichment analysis revealed that the green module genes were significantly enriched in biological processes and pathways related to mitochondrial function and energy metabolism. Consistently, almost all the hub genes of the green module ( $S L C 25 A 3, A C O 2$, $A T P 5 A 1, D L D$, and $U Q C R C 1$ ) were found to have involved in mitochondrial functions, such as the TCA cycle and ATP synthesis. Mitochondria have been discovered to play a multifunctional role in the pathogenesis of cancers(Sajnani et al. 2017). The well-known "Warburg effect" describes the shift in energy metabolism of cancer cells from mitochondrial respiration to glycolysis(Warburg 1956). This shift may result from the mutation or dysregulation of genes related to energy metabolism, such as the genes encoding the enzymes involved in the TCA cycle. A decreased copy number of mtDNA has been found in lung cancer, liver cancer, gastric cancer, and CRC(Lee et al. 2005). That is to say, the downregulated hub genes related to 
297

298

299

300

301

302

303

304

305

306

307

308

309

310

311

312

313

314

315

316

317

318

319

320

321

322

323

324

325

326

327

328

329

330

331

332

333

334

335

336

mitochondrial respiration during UC inflammation may contribute to the transformation from UC to cancer by participating in the shift of energy metabolism. In addition, the alterations in these hub genes may lead to abnormal enzymatic reactions and oncometabolites, which in turn exert carcinogenic effects mainly through epigenetic regulation(Nowicki \& Gottlieb 2015). Another hub gene of the green module, AIFM1, is a well-known caspase-independent death effector released from mitochondria, and was later discovered to also play a role in oxidative phosphorylation (OXPHOS) by regulating complex I proteins post-transcriptionally. Both cell death and OXPHOS processes have been involved in cancer pathogenesis(Liu et al. 2018; Rao et al. 2019), but the role of AIFM1 during UC-associated carcinogenesis needs further investigation.

TFE3 is a member of the MiT family of helix-loop-helix leucine zipper transcription factors that regulate their target genes by binding to E-box sequences in promoters. E-box sequences are mainly found in genes related to energy metabolism, including those involved in glycolysis and lipid metabolism. TFE3 has been reported to regulate mitochondrial dynamics and function in the liver(Pastore et al. 2017). Moreover, TFE3 has been validated as an oncogene in kidney and pancreatic cancers by virtue of its regulation of metabolic and non-metabolic pathways (Linehan et al. 2019; Perera et al. 2015). In the present study, single-gene GSEA revealed a link between TFE3 and mitochondrial function, while the latter was considered to be highly involved in UCassociated carcinogenesis. Therefore, it is logical to speculate that TFE3 may play a role in UCassociated carcinogenesis by regulating mitochondrial function. Further experimentation is required to confirm this hypothesis.

The genes encoding for proteins involved in cell-cell junctions and immune responses along with those involved in pathways, including the Hippo signaling pathway, Notch signaling pathway, and ErbB signaling pathway, were significantly enriched in the blue module. Cell-cell junctions are vital for tissue homeostasis as they not only maintain the barrier function, but regulate complex cellular signaling networks related to cell proliferation and migration(Garcia et al. 2018). Disruption of tight junctions and subsequent immune dysfunction due to exposure to antigens play critical roles in UC pathogenesis. The Hippo pathway is an important signaling pathway regulating cell proliferation and apoptosis, and its dysregulation contributes to cancers. Moreover, the activity of the Hippo pathway is highly dependent on cell junctions(Karaman \& Halder 2018). Therefore, it is logical to think that the altered cell junctions and relevant signaling network during the inflammatory phase of UC may contribute to carcinogenesis(Feigin \& Muthuswamy 2009).

The obvious limitation of the present study is the small sample size; however, additional CAD or UC-CRC clinical specimens were not available for validation. The same expression trend of hub genes in another UC dataset and the reported relationships between these genes and cancers may help in demonstrating the reliability of this study. Further efforts are being made in this direction. This work is expected to provide new insights into the process of UC-associated carcinogenesis. 


\section{Conclusions}

338 In conclusion, this study, using WGCNA, found two gene modules that were significantly 339 correlated with the process of UC-associated carcinogenesis from inflammation to dysplasia.

340 From these two modules, 13 hub genes (SLC25A3, ACO2, AIFM1, ATP5A1, DLD, TFE3,

341 UQCRC1, ADIPOR2, SLC35D1, TOR1AIP1, PRR5L, ATOX1, and DTX3) were identified as key

342 genes involved in UC-associated carcinogenesis. Functional enrichment analysis revealed the

343 critical biological processes contributing to UC-associated carcinogenesis, mainly include

344 mitochondrial dysfunction, cell-cell junction, and immune responses. TFE3, a transcription

345 factor related to mitochondrial function and cancer, seem to play a central role in this process.

346

\section{Acknowledgements}

348 We would like to thank Editage (www.editage.cn) for English language editing.

349

350

351

352

353

354

355

356

357

358

359

360

361

362

363

364

365

366

367

368

369

370

371

372

373

374

375

376

377

378

379

380

381

382

\section{References}

Beckmann H, Su LK, and Kadesch T. 1990. TFE3: a helix-loop-helix protein that activates transcription through the immunoglobulin enhancer muE3 motif. Genes Dev 4:167-179.

Bernstein CN, Blanchard JF, Kliewer E, and Wajda A. 2001. Cancer risk in patients with inflammatory bowel disease: a population-based study. Cancer 91:854-862.

Bjerrum JT, Nielsen OH, Riis LB, Pittet V, Mueller C, Rogler G, and Olsen J. 2014. Transcriptional analysis of left-sided colitis, pancolitis, and ulcerative colitis-associated dysplasia. Inflamm Bowel Dis 20:2340-2352.

Bopanna S, Ananthakrishnan AN, Kedia S, Yajnik V, and Ahuja V. 2017. Risk of colorectal cancer in Asian patients with ulcerative colitis: a systematic review and meta-analysis. Lancet Gastroenterol Hepatol 2:269-276.

Bruggemann M, Gromes A, Poss M, Schmidt D, Klumper N, Tolkach Y, Dietrich D, Kristiansen G, Muller SC, and Ellinger J. 2017. Systematic Analysis of the Expression of the Mitochondrial ATP Synthase (Complex V) Subunits in Clear Cell Renal Cell Carcinoma. Transl Oncol 10:661-668.

Byeon JS, Jeong JY, Kim MJ, Lee SM, Nam WH, Myung SJ, Kim JG, Yang SK, Kim JH, and Suh DJ. 2010. Adiponectin and adiponectin receptor in relation to colorectal cancer progression. Int J Cancer 127:2758-2767.

Chung SJ, Nagaraju GP, Nagalingam A, Muniraj N, Kuppusamy P, Walker A, Woo J, Gyorffy B, Gabrielson E, Saxena NK et al. . 2017. ADIPOQ/adiponectin induces cytotoxic autophagy in breast cancer cells through STK11/LKB1-mediated activation of the AMPKULK1 axis. Autophagy 13:1386-1403.

Ciccarone F, Di Leo L, Lazzarino G, Maulucci G, Di Giacinto F, Tavazzi B, and Ciriolo MR. 2020. Aconitase 2 inhibits the proliferation of MCF-7 cells promoting mitochondrial oxidative metabolism and ROS/FoxO1-mediated autophagic response. $\mathrm{Br} \mathrm{J}$ Cancer 122:182-193.

Ding XY, Hu HY, Huang KN, Wei RQ, Min J, Qi C, Tang H, and Qin X. 2020. Ubiquitination of NOTCH2 by DTX3 suppresses the proliferation and migration of human esophageal carcinoma. Cancer Sci 111:489-501.

Feigin ME, and Muthuswamy SK. 2009. Polarity proteins regulate mammalian cell-cell junctions and cancer pathogenesis. Curr Opin Cell Biol 21:694-700.

Garcia MA, Nelson WJ, and Chavez N. 2018. Cell-Cell Junctions Organize Structural and Signaling Networks. Cold Spring Harb Perspect Biol 10. 
383

384

385

386

387

388

389

390

391

392

393

394

395

396

397

398

399

400

401

402

403

404

405

406

407

408

409

410

411

412

413

414

415

416

417

418

419

420

421

422

423

424

425

426

427

428

429

430

431

432

Itzkowitz SH. 2006. Molecular biology of dysplasia and cancer in inflammatory bowel disease. Gastroenterol Clin North Am 35:553-571.

Itzkowitz SH, and Yio X. 2004. Inflammation and cancer IV. Colorectal cancer in inflammatory bowel disease: the role of inflammation. Am J Physiol Gastrointest Liver Physiol 287:G717.

Jana A, Das A, Krett NL, Guzman G, Thomas A, Mancinelli G, Bauer J, Ushio-Fukai M, Fukai T, and Jung B. 2020. Nuclear translocation of Atox1 potentiates activin A-induced cell migration and colony formation in colon cancer. PLoS One 15:e0227916.

Ju Z, Bhardwaj A, Embury MD, Singh H, Gunaratne PH, Bedrosian I, and Wang J. 2020. Integrative Analyses of Multilevel Omics Reveal Preneoplastic Breast to Possess a Molecular Landscape That is Globally Shared with Invasive Basal-Like Breast Cancer (Running Title: Molecular Landscape of Basal-Like Breast Cancer Progression). Cancers (Basel) 12.

Karaman R, and Halder G. 2018. Cell Junctions in Hippo Signaling. Cold Spring Harb Perspect Biol 10.

Karginova O, Weekley CM, Raoul A, Alsayed A, Wu T, Lee SS, He C, and Olopade OI. 2019. Inhibition of Copper Transport Induces Apoptosis in Triple-Negative Breast Cancer Cells and Suppresses Tumor Angiogenesis. Mol Cancer Ther 18:873-885.

Khalil AA. 2007. Biomarker discovery: a proteomic approach for brain cancer profiling. Cancer Sci 98:201-213.

Kim YJ, Bond GJ, Tsang T, Posimo JM, Busino L, and Brady DC. 2019. Copper chaperone ATOX1 is required for MAPK signaling and growth in BRAF mutation-positive melanoma. Metallomics 11:1430-1440.

Laiho P, Hienonen T, Mecklin JP, Jarvinen H, Karhu A, Launonen V, and Aaltonen LA. 2003. Mutation and LOH analysis of ACO2 in colorectal cancer: no evidence of biallelic genetic inactivation. J Med Genet 40:e73.

Laine L, Kaltenbach T, Barkun A, McQuaid KR, Subramanian V, Soetikno R, and Panel SGD. 2015. SCENIC international consensus statement on surveillance and management of dysplasia in inflammatory bowel disease. Gastroenterology 148:639-651 e628.

Langfelder P, and Horvath S. 2008. WGCNA: an R package for weighted correlation network analysis. BMC Bioinformatics 9:559.

Lee HC, Yin PH, Lin JC, Wu CC, Chen CY, Wu CW, Chi CW, Tam TN, and Wei YH. 2005. Mitochondrial genome instability and mtDNA depletion in human cancers. Ann N Y Acad Sci 1042:109-122.

Li K, Strauss R, Ouahed J, Chan D, Telesco SE, Shouval DS, Canavan JB, Brodmerkel C, Snapper SB, and Friedman JR. 2018. Molecular Comparison of Adult and Pediatric Ulcerative Colitis Indicates Broad Similarity of Molecular Pathways in Disease Tissue. J Pediatr Gastroenterol Nutr 67:45-52.

Li W, Wubulikasimu G, Zhao X, Wang C, Liu R, Wang L, Zhu X, and Chen Z. 2019. UQCRC1 downregulation is correlated with lymph node metastasis and poor prognosis in CRC. Eur J Surg Oncol 45:1005-1010.

Linehan WM, Schmidt LS, Crooks DR, Wei D, Srinivasan R, Lang M, and Ricketts CJ. 2019. The Metabolic Basis of Kidney Cancer. Cancer Discov 9:1006-1021.

Liu D, Liu M, Wang W, Pang L, Wang Z, Yuan C, and Liu K. 2018. Overexpression of apoptosisinducing factor mitochondrion-associated 1 (AIFM1) induces apoptosis by promoting the transcription of caspase 3 and DRAM in hepatoma cells. Biochem Biophys Res Commun 498:453-457.

Millan A, and Huerta S. 2009. Apoptosis-inducing factor and colon cancer. J Surg Res 151:163170.

Nowicki S, and Gottlieb E. 2015. Oncometabolites: tailoring our genes. FEBS J 282:2796-2805.

Peer) reviewing PDF | (2020:12:56299:2:0:NEW 25 Mar 2021) 
433

434

435

436

437

438

439

440

441

442

443

444

445

446

447

448

449

450

451

452

453

454

455

456

457

458

459

460

461

462

463

464

465

466

467

468

469

470

471

472

473

474

475

476

477

478

479

480

481

482

483

Oehler VG, Yeung KY, Choi YE, Bumgarner RE, Raftery AE, and Radich JP. 2009. The derivation of diagnostic markers of chronic myeloid leukemia progression from microarray data. Blood 114:3292-3298.

Pastore N, Vainshtein A, Klisch TJ, Armani A, Huynh T, Herz NJ, Polishchuk EV, Sandri M, and Ballabio A. 2017. TFE3 regulates whole-body energy metabolism in cooperation with TFEB. EMBO Mol Med 9:605-621.

Perera RM, Stoykova S, Nicolay BN, Ross KN, Fitamant J, Boukhali M, Lengrand J, Deshpande V, Selig MK, Ferrone CR et al. . 2015. Transcriptional control of autophagy-lysosome function drives pancreatic cancer metabolism. Nature 524:361-365.

Rao S, Mondragon L, Pranjic B, Hanada T, Stoll G, Kocher T, Zhang P, Jais A, Lercher A, Bergthaler A et al. . 2019. AlF-regulated oxidative phosphorylation supports lung cancer development. Cell Res 29:579-591.

Ritchie ME, Phipson B, Wu D, Hu Y, Law CW, Shi W, and Smyth GK. 2015. limma powers differential expression analyses for RNA-sequencing and microarray studies. Nucleic Acids Res 43:e47.

Rubin DT, Ananthakrishnan AN, Siegel CA, Sauer BG, and Long MD. 2019. ACG Clinical Guideline: Ulcerative Colitis in Adults. Am J Gastroenterol 114:384-413.

Sajnani K, Islam F, Smith RA, Gopalan V, and Lam AK. 2017. Genetic alterations in Krebs cycle and its impact on cancer pathogenesis. Biochimie 135:164-172.

Seth R, Keeley J, Abu-Ali G, Crook S, Jackson D, and llyas M. 2009. The putative tumour modifier gene ATP5A1 is not mutated in human colorectal cancer cell lines but expression levels correlate with TP53 mutations and chromosomal instability. J Clin Pathol 62:598-603.

Subramanian A, Tamayo P, Mootha VK, Mukherjee S, Ebert BL, Gillette MA, Paulovich A, Pomeroy SL, Golub TR, Lander ES et al. . 2005. Gene set enrichment analysis: a knowledge-based approach for interpreting genome-wide expression profiles. Proc Natl Acad Sci U S A 102:15545-15550.

Tang A, Li N, Li X, Yang H, Wang W, Zhang L, Li G, Xiong W, Ma J, and Shen S. 2012. Dynamic activation of the key pathways: linking colitis to colorectal cancer in a mouse model. Carcinogenesis 33:1375-1383.

Thedieck K, Polak P, Kim ML, Molle KD, Cohen A, Jeno P, Arrieumerlou C, and Hall MN. 2007. PRAS40 and PRR5-like protein are new mTOR interactors that regulate apoptosis. PLoS One 2:e1217.

Viola M, Bruggemann K, Karousou E, Caon I, Carava E, Vigetti D, Greve B, Stock C, De Luca G, Passi A et al. . 2017. MDA-MB-231 breast cancer cell viability, motility and matrix adhesion are regulated by a complex interplay of heparan sulfate, chondroitin-/dermatan sulfate and hyaluronan biosynthesis. Glycoconj J 34:411-420.

Wang P, Mai C, Wei YL, Zhao JJ, Hu YM, Zeng ZL, Yang J, Lu WH, Xu RH, and Huang P. 2013. Decreased expression of the mitochondrial metabolic enzyme aconitase (ACO2) is associated with poor prognosis in gastric cancer. Med Oncol 30:552.

Wang Q, Li M, Gan Y, Jiang S, Qiao J, Zhang W, Fan Y, Shen Y, Song Y, Meng Z et al. . 2020. Mitochondrial Protein UQCRC1 is Oncogenic and a Potential Therapeutic Target for Pancreatic Cancer. Theranostics 10:2141-2157.

Warburg O. 1956. On the origin of cancer cells. Science 123:309-314.

Willett R, Martina JA, Zewe JP, Wills R, Hammond GRV, and Puertollano R. 2017. TFEB regulates lysosomal positioning by modulating TMEM55B expression and JIP4 recruitment to lysosomes. Nat Commun 8:1580.

Yu G, Wang LG, Han Y, and He QY. 2012. clusterProfiler: an R package for comparing biological themes among gene clusters. OMICS 16:284-287.

Zhang B, and Horvath S. 2005. A general framework for weighted gene co-expression network analysis. Stat Appl Genet Mol Biol 4:Article17.

Peer) reviewing PDF | (2020:12:56299:2:0:NEW 25 Mar 2021) 
484

485

486

487

488

489

490

491
Zhang Y, Wu X, Zhang C, Wang J, Fei G, Di X, Lu X, Feng L, Cheng S, and Yang A. 2020. Dissecting expression profiles of gastric precancerous lesions and early gastric cancer to explore crucial molecules in intestinal-type gastric cancer tumorigenesis. J Pathol 251:135-146.

Zou D, Li R, Huang X, Chen G, Liu Y, Meng Y, Wang Y, Wu Y, and Mao Y. 2019. Identification of molecular correlations of RBM8A with autophagy in Alzheimer's disease. Aging (Albany NY) 11:11673-11685. 
Figure 1

Overview of the work flow and GEO data.

(A) Flow chart of the present study; (B) Principal component analysis plot of the gene expression profiles in GSE47908. Two principal components containing $37.23 \%$ of the variance illustrate separate clustering of the three group of samples; (C) Principal component analysis plot of the gene expression profiles in GSE87466. It shows the separate clustering of the two groups with a few samples overlapping by two principal component containing $31.81 \%$ of the variance.

A

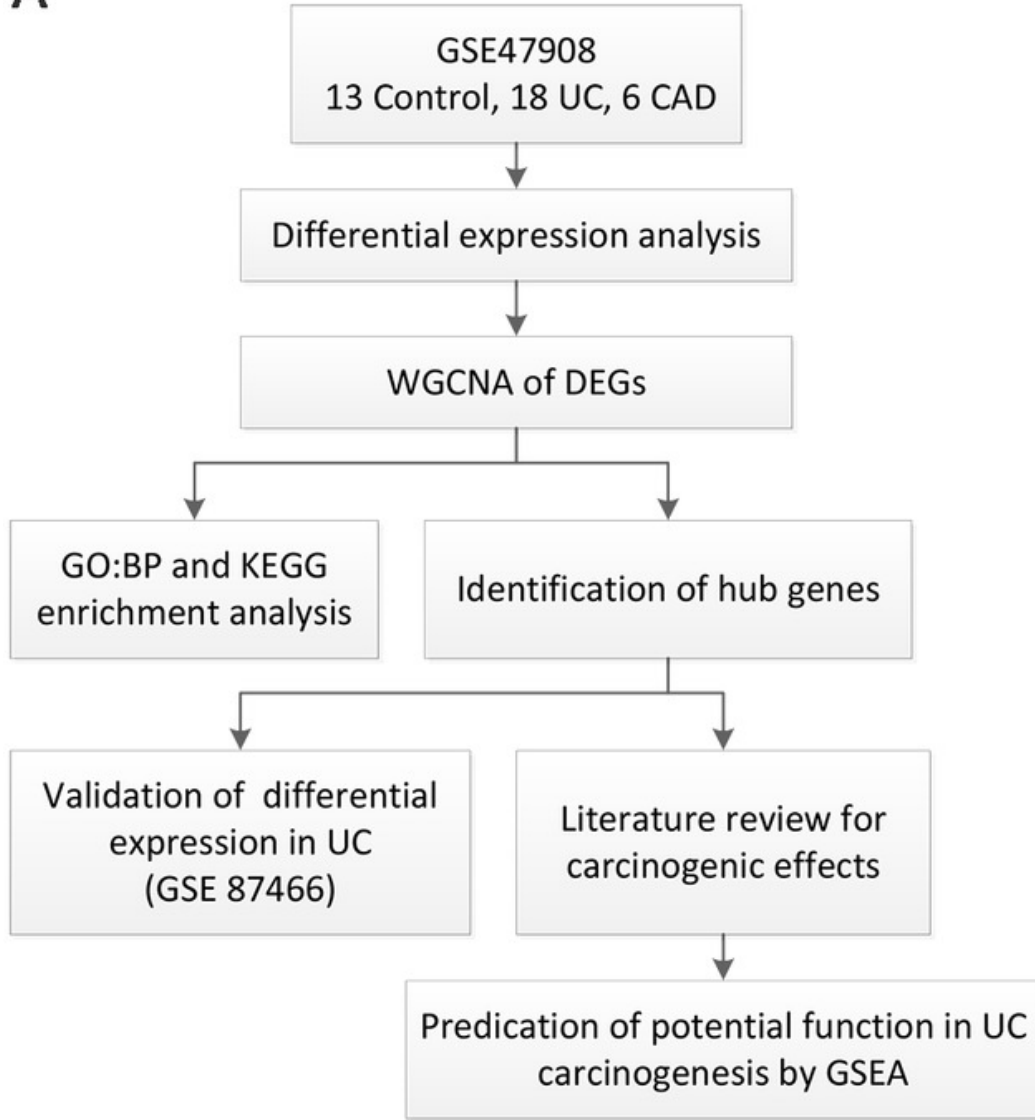

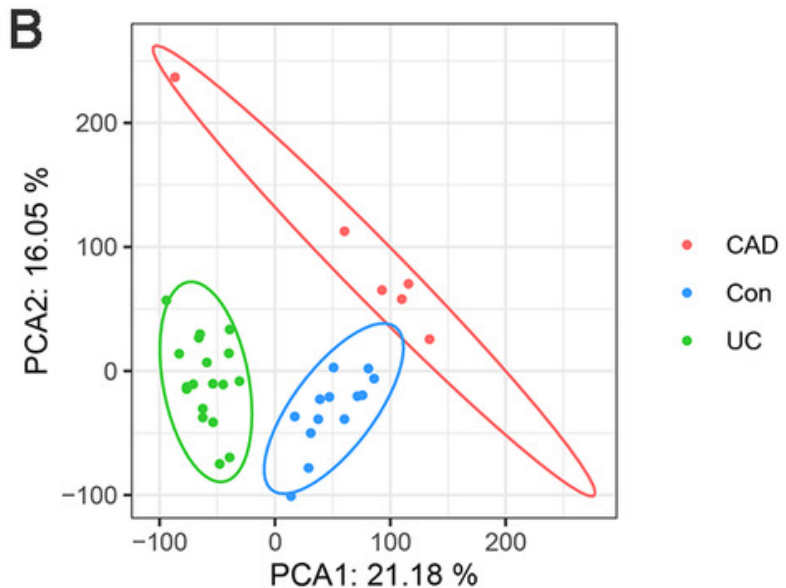

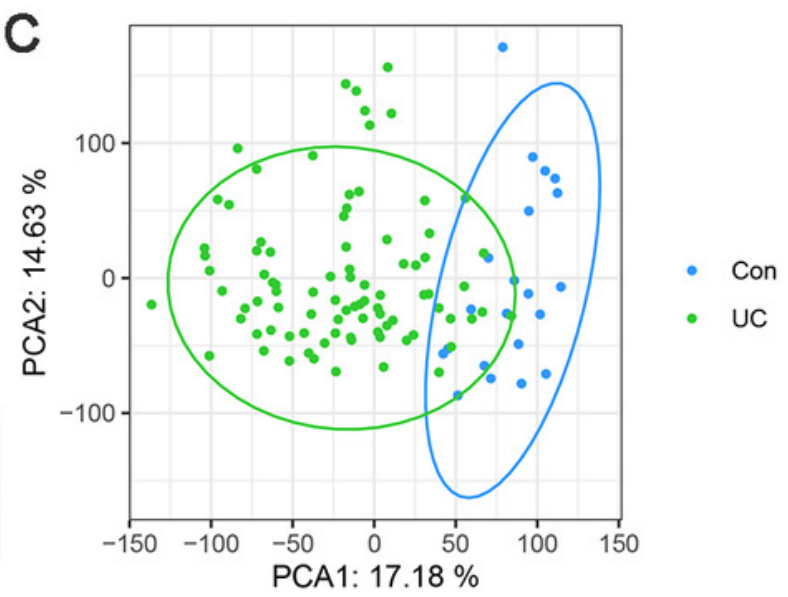




\section{Figure 2}

Differential expression analysis.

(A) Volcano plot between UC and control groups. There are 679 up-regulated genes and 288 down-regulated genes in UC compared to controls when $\log _{2} \mathrm{FC}$ cut-off value was set to 1.092 by calculated. (B) Volcano plot between CAD and UC groups. There are 260 up-regulated genes and 616 down-regulated genes in CAD compared to UC when $\log _{2} \mathrm{FC}$ cut-off value was set to 1.073 by calculated. (C) Volcano plot between CAD and control groups. Black vertical line indicates $\log _{2} \mathrm{FC}$ of 0.804 , and grey vertical indicates $\log _{2} \mathrm{FC}$ of 0.3 . There are 305 upregulated genes and 462 down-regulated genes in CAD compared to controls when $\log _{2} \mathrm{FC}$ cut-off value was set to $0.804,2904$ up-regulated genes and 2025 down-regulated genes when $\log _{2} \mathrm{FC}$ cut-off value was set to 0.3 . (D) Venn diagram illustrating up-regulated genes in different groups. (E) Venn diagram illustrating down-regulated genes in different groups. (F) Heat map of top 40 up-regulated and 40 down-regulated DEGs between CAD and controls after sorted by adjusted $p$-value from smallest to largest. 

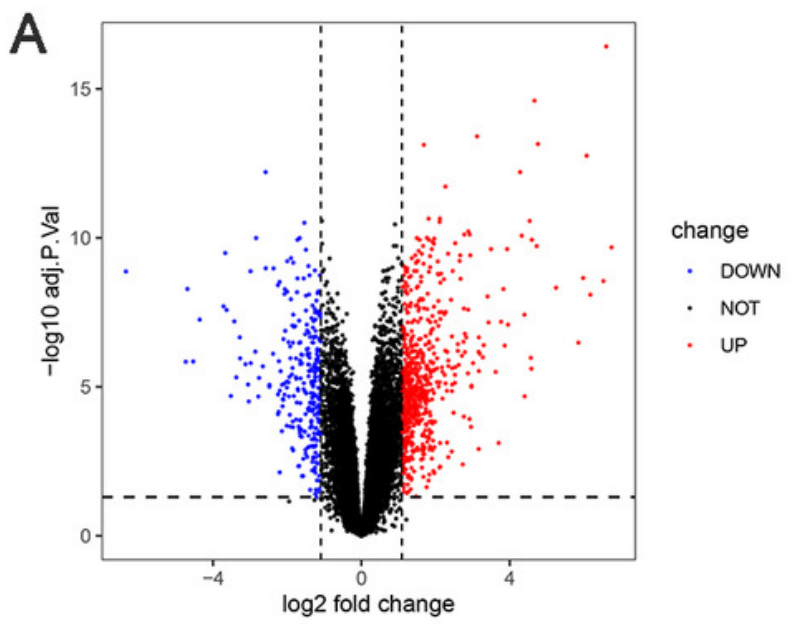

B

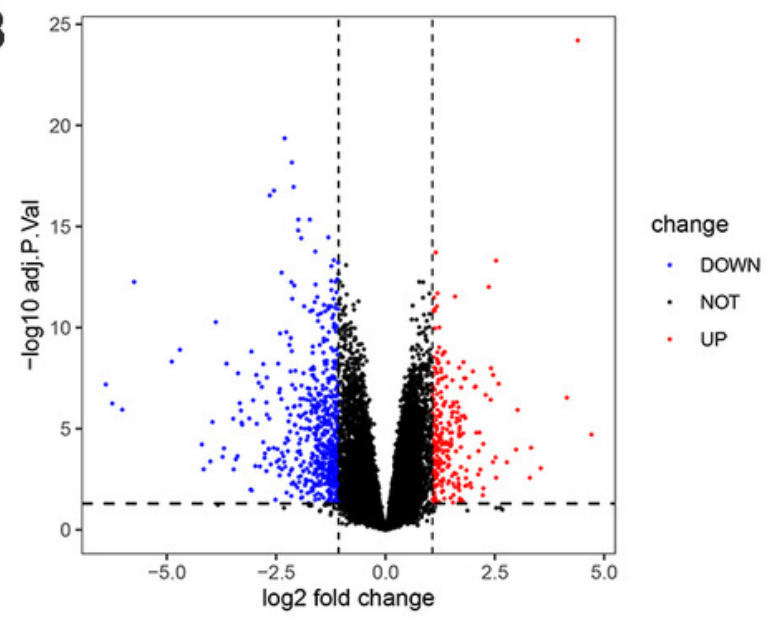

C

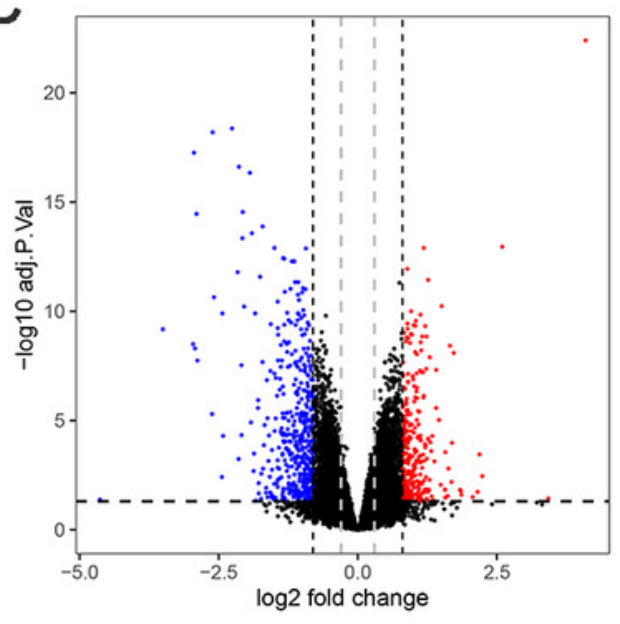

D

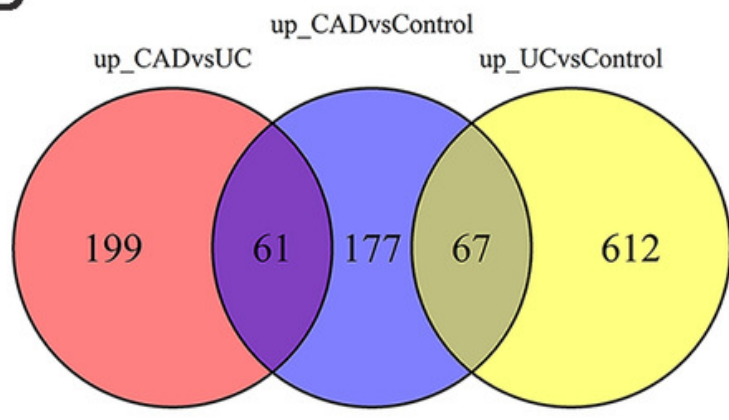

E

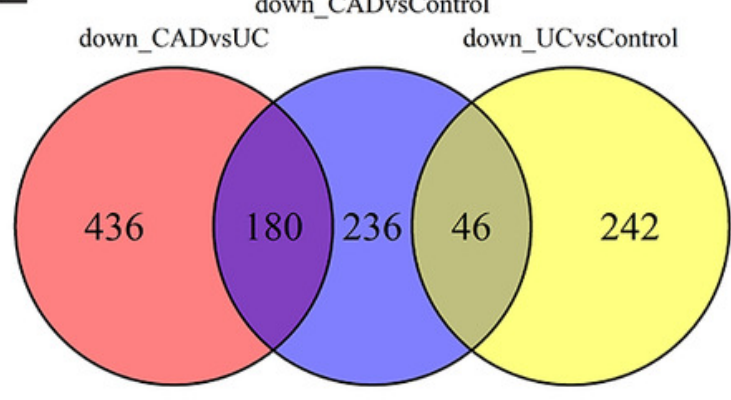

change

- DOWN

- NOT

- UP

F
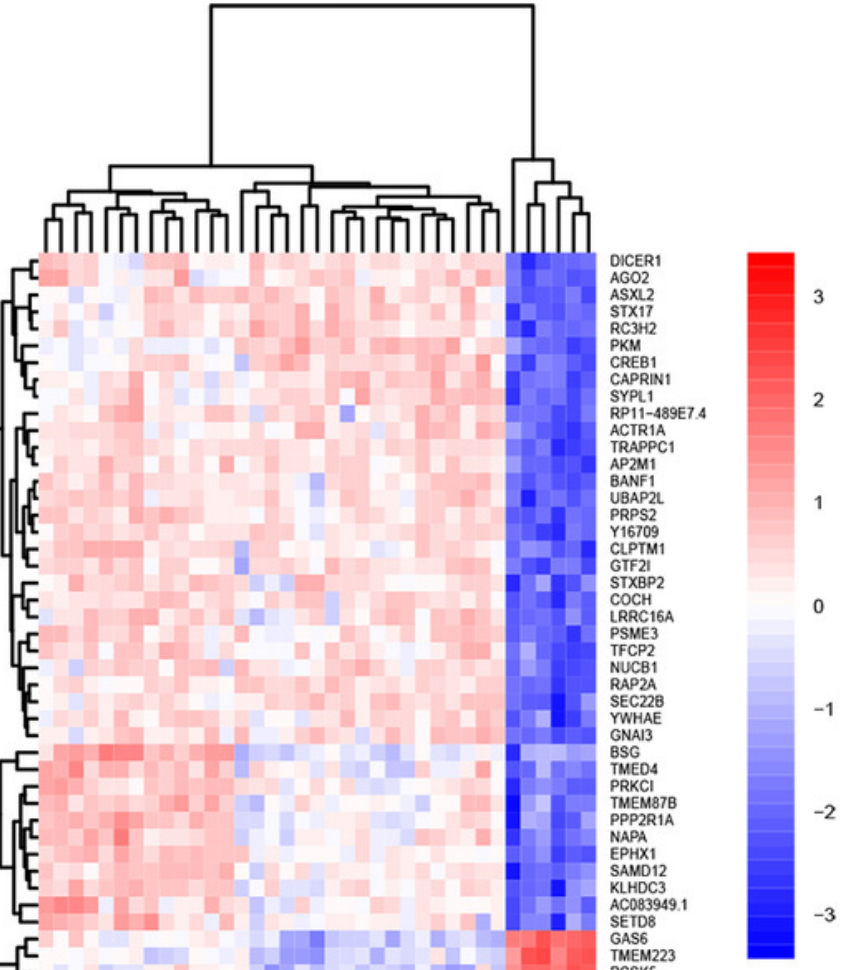

RP11-650K20.3

PABPC3 3

GS1-111G1

DNAJCA
BPTF

BPTF
LOC100996255

ASB8

BRI3
EIV6

ZNF688

MSPA

DVL2

MGC12488

RP11-45210.1

MAFG

EAFG

BBSA

Z8ED6

PARP10

TCIRG

FLASP1

LIGL1

AASS

C19ort60

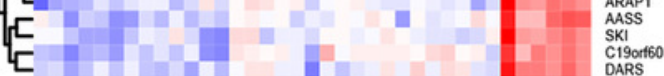

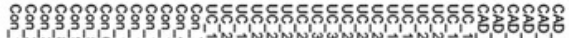




\section{Figure 3}

Weighted gene co-expression network analysis.

(A) Network topology for different soft-thresholding powers. The scale-free topology can be attained at the soft-thresholding power of 12. (B) Cluster dendrogram and gene modules identified by WGCNA. (C) Correlation heat map of gene modules and traits, labelled with correlation coefficient and $p$ value. The trait "Progress" means the progressive process from normal to UC, and then to CAD, which is assigned the value of 0,1 and 2, to represent the control, UC and CAD group, respectively. 


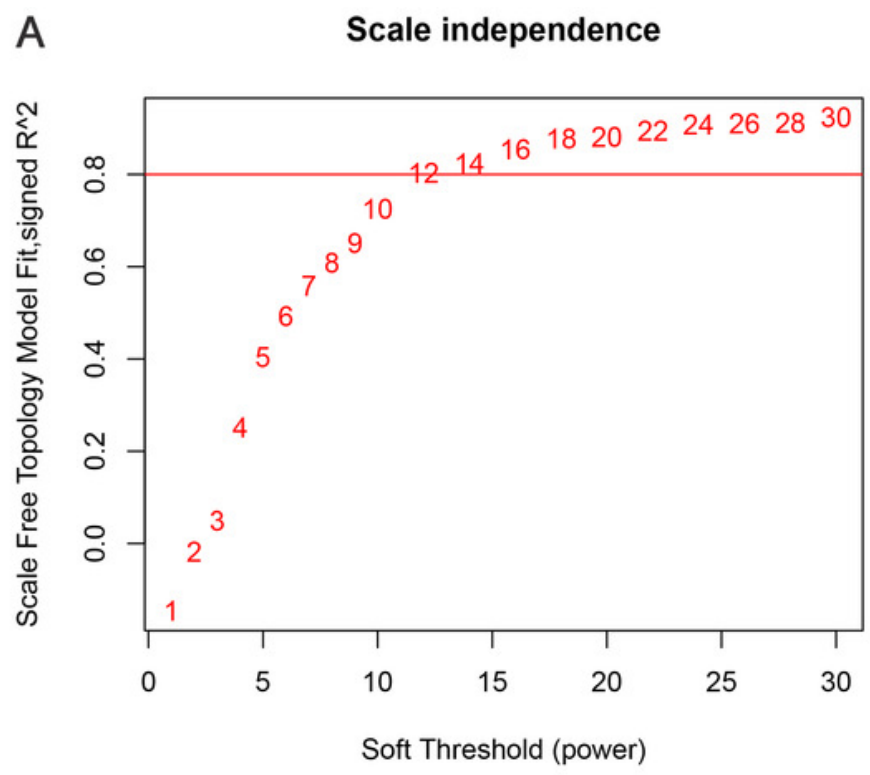

C

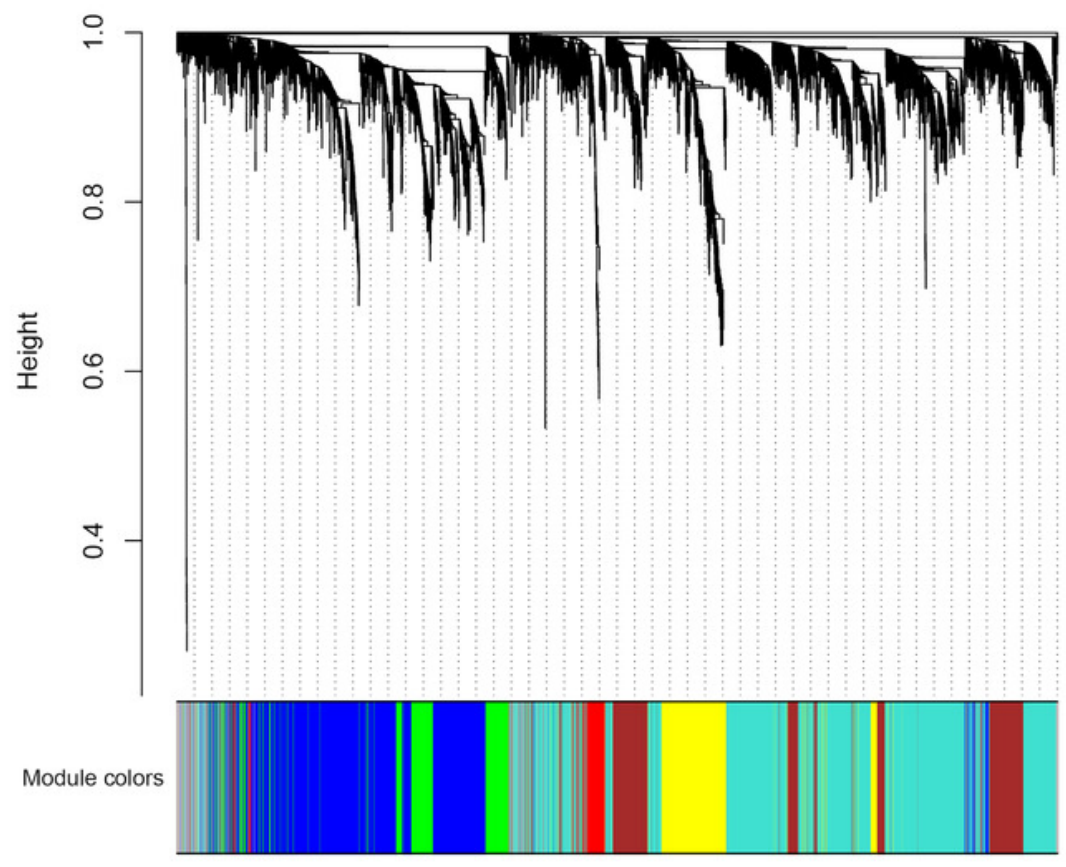

B

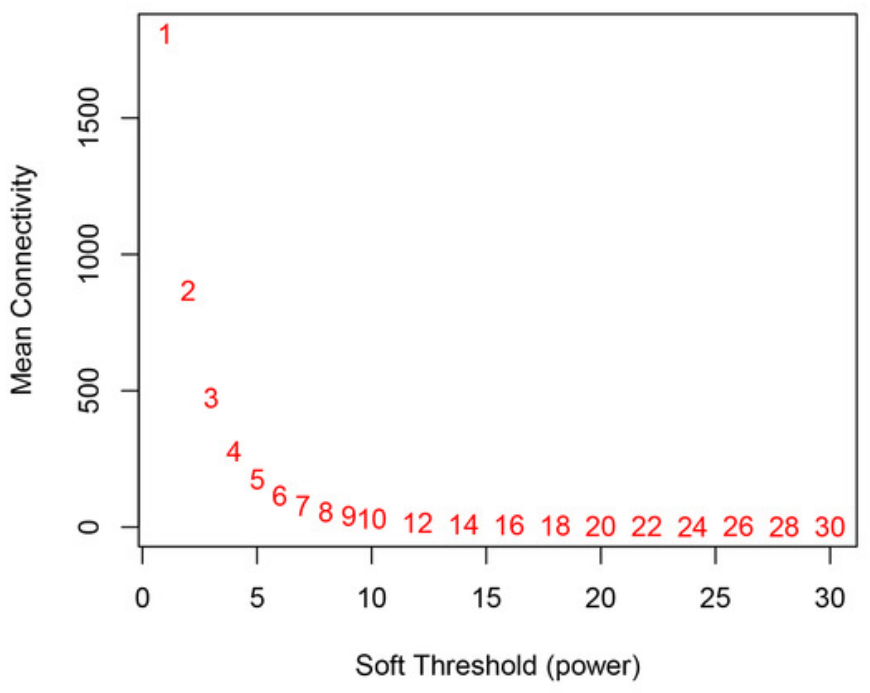

D Module-trait relationships

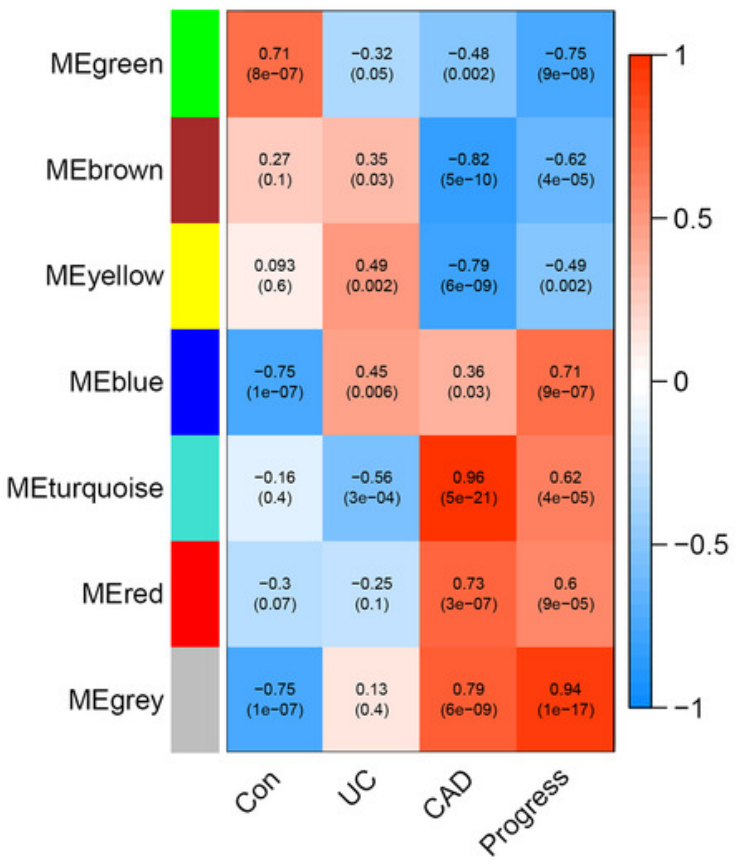


Figure 4

GO-BP and KEGG pathways enrichment analysis.

(A) Biological processes enriched in green module genes. (B) KEGG pathways enriched in green module genes. (C) Biological processes enriched in blue module genes. (D) KEGG pathways enriched in blue module genes.

A

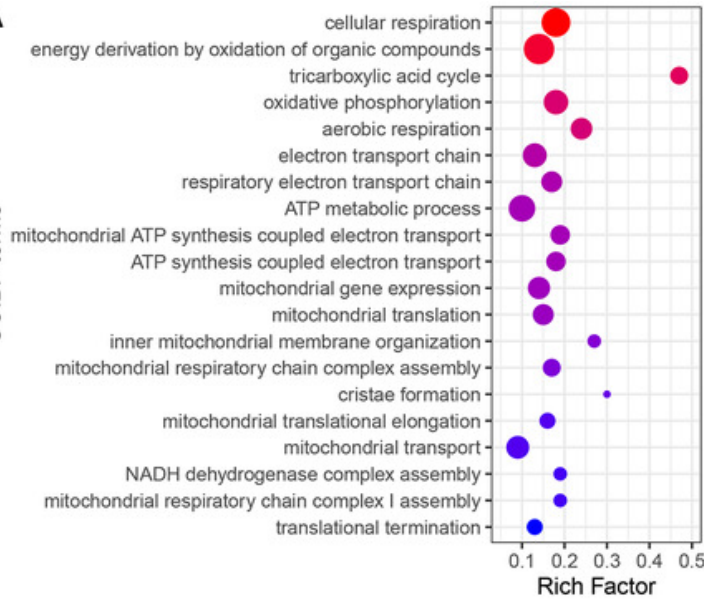

C

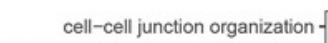
protein extracellular matrix organization extracellular matrix organization
extracellular structure organization extracellular structure organization epidermal growth factor receptor signaling pathway cell-cell junction assembly

है

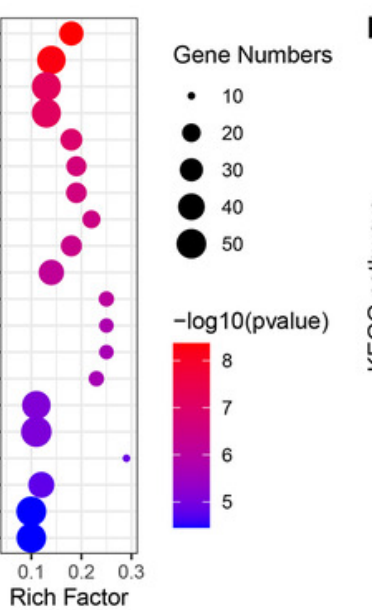

Rich Factor $-\log 10$ (pvalue)

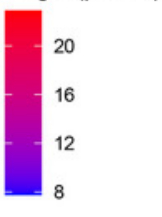

Gene Numbers

- 10

20

30

D
B

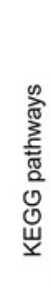

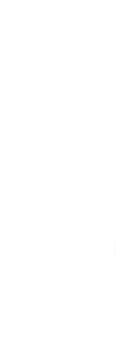

Citrate cycle (TCA cycle) Oxidative phosphorylation

Parkinson disease -

Thermogenesis -

Huntington disease Prion disease

Non-alcoholic fatty liver disease 2-Oxocarboxylic acid metabolism Alzheimer disease Amyotrophic lateral sclerosis Biosynthesis of amino acids.

Propanoate metabolism thways of neurodegeneration - multiple diseases Pyruvate metabolism Glyoxylate and dicarboxylate metabolism Arginine biosynthesis Terpenoid backbone biosynthesis Valine, leucine and isoleucine degradation Arginine and proline metabolism

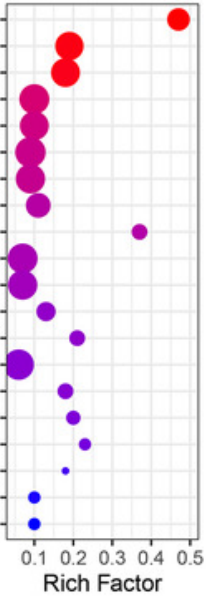

Gene Numbers

- 5

- 10

- 15

20

25

$-\log 10$ (pvalue)

10

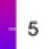

Glycosaminoglycan hiosynthesis - chondrotin sulate / dermatan Cell adhesion molecules ErbB signaling pathway epithelial cell morphogenesis epithelial tube morphogenesis neutrophil activation neutrophil degranulation
Human papillomavirus infection Focal adhesion -
Tight junction ECM-receptor interaction Epithelial cell signaling in Helicobacter pylori infection Growth hormone synthesis, secretion and action Hippo signaling pathway - multiple species Notch signaling pathway Leukocyte transendothelial migration Thyroid hormone synthesis GnRH signaling pathway Relaxin signaling pathway Chronic myeloid leukemia

Glycosaminoglycan biosynthesis - chondroitin sulfate / dermatan sulfate Longevity regulating pathway Endometrial cancer Endocytosis -

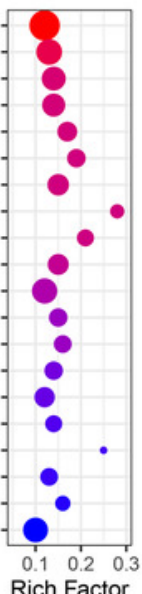

Gene Numbers

- 10

20

30

40

$-\log 10$ (pvalue)

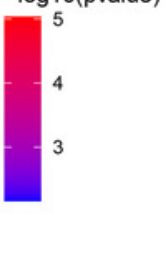


Figure 5

Hub genes of green and blue modules.

(A) Identification of hub genes of green module by MM and GS filtering. (B) Differential expression patterns of 7 hub genes of green module. (C) Identification of hub genes of blue module by MM and GS filtering. (D) Differential expression patterns of 6 hub genes of blue module.

A

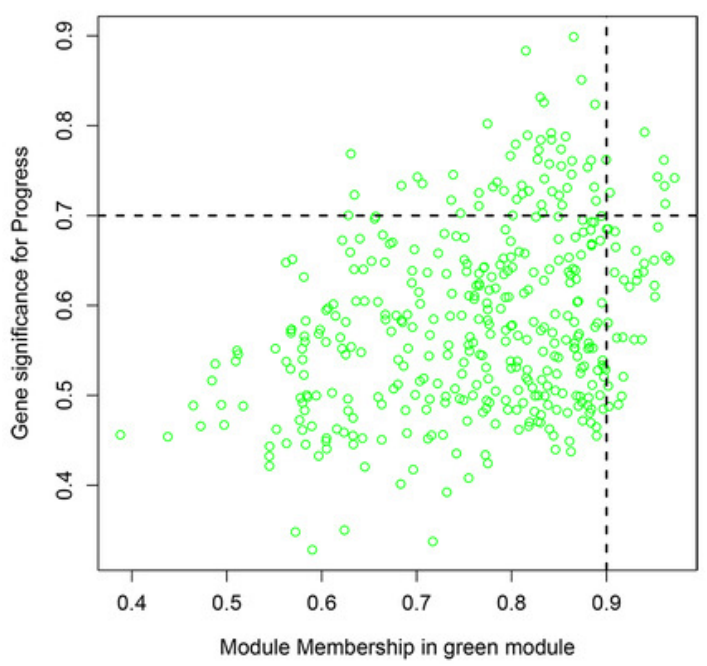

C

Module membership vs. gene significance cor $=0.23, p=1.7 e-16$

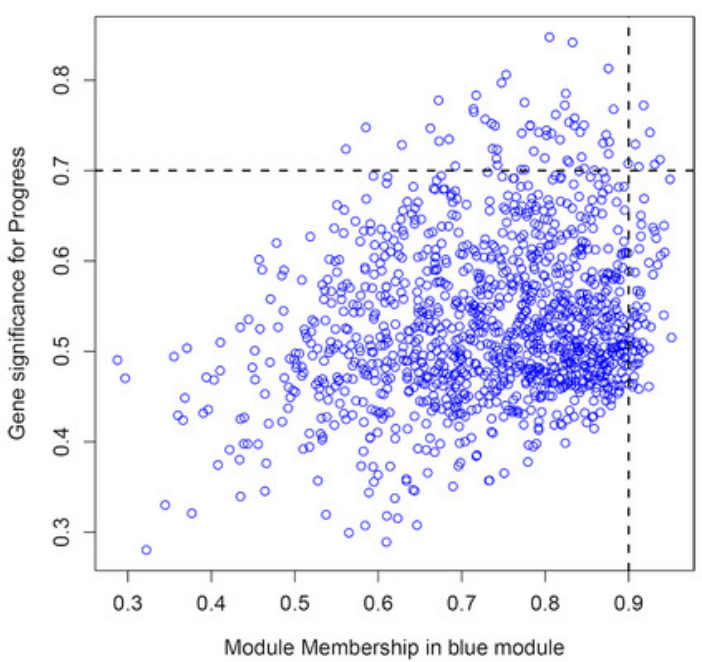

B

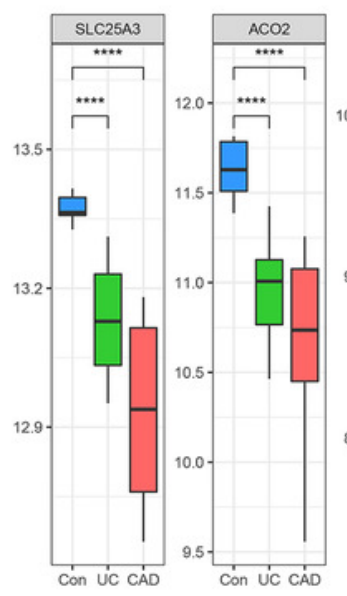

D

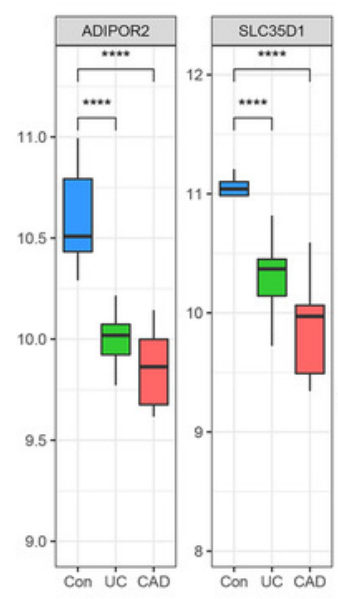

Seven hub genes of green module
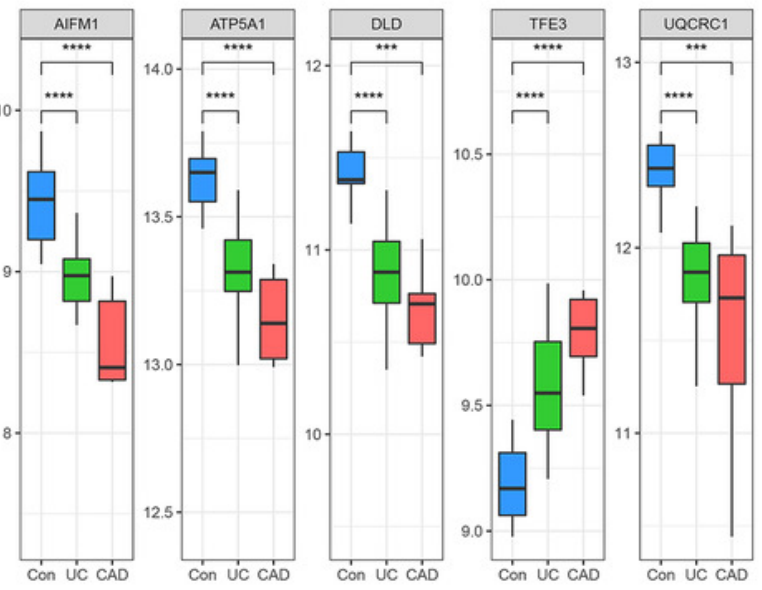

Six hub genes of blue module
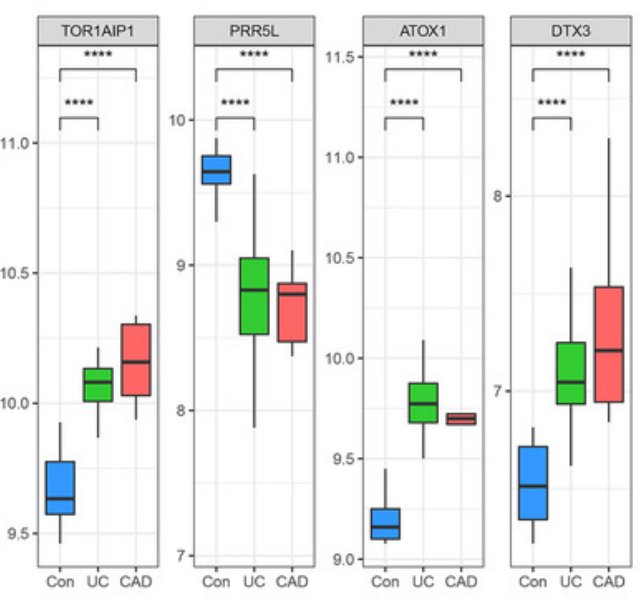

group

追 con

官 Uc

官 CAD 
Figure 6

Validation of the expression change of hub genes in UC from GSE87466. 

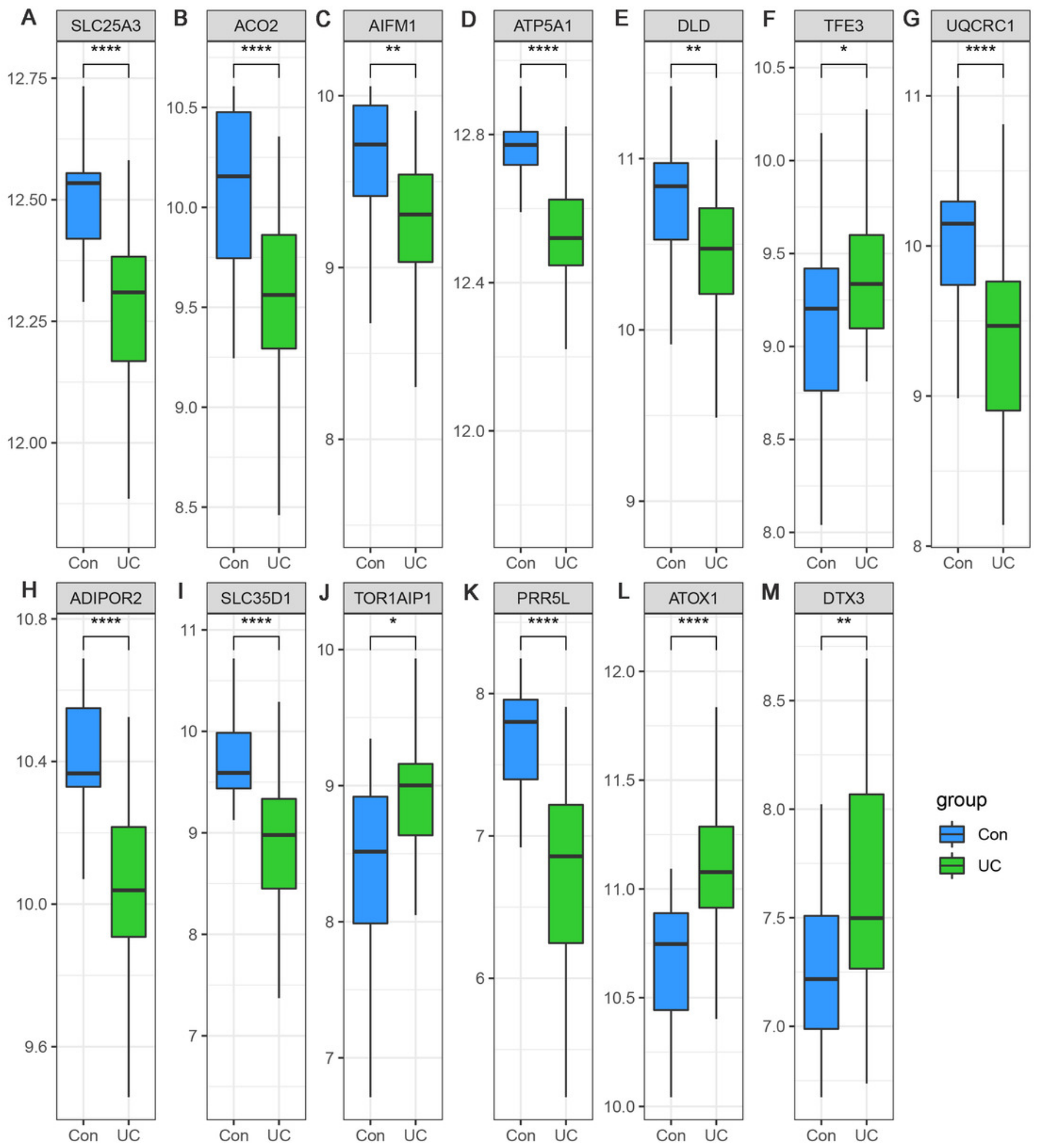
Figure 7

Single-gene GSEA analysis of TFE3.

(A) Enriched biological processes of genes positively correlated with TFE3. (B) Enriched biological processes of genes negatively correlated with TFE3. (C) Enriched KEGG pathways positively correlated with TFE3. (D) Enriched KEGG pathways negatively correlated with TFE3.
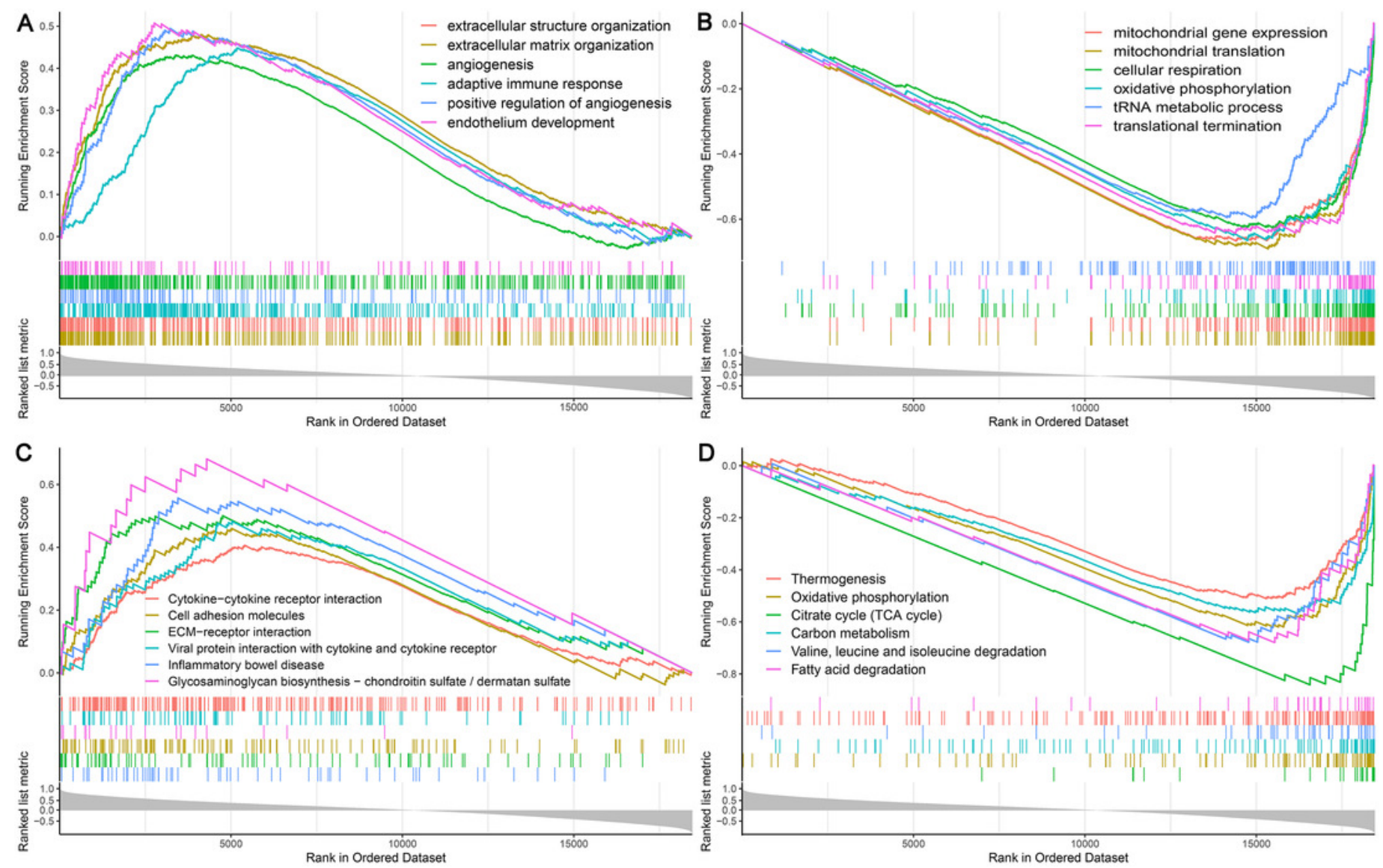


\section{Table 1 (on next page)}

Literature review of the proof for the carcinogenic effects of hub genes. 
Table 1. Literature review of the proof for the carcinogenic effects of hub genes.

\begin{tabular}{|c|c|c|c|c|}
\hline Genes & Encoding protein & Known function & $\begin{array}{l}\text { Any proof for } \\
\text { carcinogenesis }\end{array}$ & $\begin{array}{l}\text { Any proof } \\
\text { for CRC }\end{array}$ \\
\hline SLC25A3 & $\begin{array}{l}\text { Phosphate carrier protein, } \\
\text { mitochondrial }\end{array}$ & $\begin{array}{l}\text { Transport of phosphate to } \\
\text { mitochondrial matrix, involve in } \\
\text { ATP synthesis }\end{array}$ & Yes ${ }^{[18]}$ & No \\
\hline $\mathrm{ACO} 2$ & $\begin{array}{l}\text { Aconitate hydratase, } \\
\text { mitochondrial }\end{array}$ & $\begin{array}{l}\text { Catalyze the isomerization of citrate } \\
\text { to isocitrate, involve in tricarboxylic } \\
\text { acid cycle }\end{array}$ & Yes $^{[19,20]}$ & $\mathrm{No}^{[21]}$ \\
\hline AIFM1 & $\begin{array}{l}\text { Apoptosis-inducing factor } 1 \text {, } \\
\text { mitochondrial }\end{array}$ & $\begin{array}{l}\text { Regulate apoptosis, and involve in } \\
\text { mitochondrial respiratory }\end{array}$ & $\mathrm{Yes}^{[22,23]}$ & $\mathrm{Yes}^{[24]}$ \\
\hline ATP5A1 & $\begin{array}{l}\text { ATP synthase subunit alpha, } \\
\text { mitochondrial }\end{array}$ & $\begin{array}{l}\text { A component of the mitochondrial } \\
\text { complex V in the respiratory chain, } \\
\text { involve in ATP synthesis }\end{array}$ & $\mathrm{Yes}^{[25]}$ & $\mathrm{Yes}^{[26]}$ \\
\hline DLD & $\begin{array}{l}\text { Dihydrolipoyl dehydrogenase, } \\
\text { mitochondrial }\end{array}$ & $\begin{array}{l}\text { Function as a dehydrogenase } \\
\text { regulating energy metabolism, or as a } \\
\text { protease }\end{array}$ & $\mathrm{Yes}^{[27]}$ & No \\
\hline TFE3 & $\begin{array}{l}\text { Transcription factor binding to } \\
\text { IGHM enhancer } 3\end{array}$ & $\begin{array}{l}\text { Function as transcription factor, } \\
\text { regulate many biological processes, } \\
\text { such as energy metabolism,lysosomal } \\
\text { biogenesis and immune response }\end{array}$ & Yes $[28,29]$ & No \\
\hline UQCRC1 & $\begin{array}{l}\text { Cytochrome b-c } 1 \text { complex } \\
\text { subunit } 1 \text {, mitochondrial }\end{array}$ & $\begin{array}{l}\text { A component of the complex III in } \\
\text { the respiratory chain, involve in ATP } \\
\text { synthesis }\end{array}$ & $\mathrm{Yes}^{[30]}$ & $\mathrm{Yes}^{[31]}$ \\
\hline ADIPOR2 & Adiponectin receptor protein 2 & $\begin{array}{l}\text { Regulates glucose and lipid } \\
\text { metabolism, mediate increased } \\
\text { AMPK and PPAR-alpha ligand } \\
\text { activities }\end{array}$ & $\mathrm{Yes}^{[32]}$ & $\mathrm{Yes}^{[33]}$ \\
\hline SLC35D1 & $\begin{array}{l}\text { UDP-glucuronic acid/UDP-N- } \\
\text { acetylgalactosamine transporter }\end{array}$ & $\begin{array}{l}\text { Transport both UDP-glucuronic acid } \\
\text { and UDP-N-acetylgalactosamine to } \\
\text { ER lumen, participate in } \\
\text { glucuronidation and/or chondroitin } \\
\text { sulfate biosynthesis }\end{array}$ & $\mathrm{Yes}^{[34]}$ & No \\
\hline TOR1AIP1 & Torsin-1A-interacting protein 1 & $\begin{array}{l}\text { Localize to the inner nuclear } \\
\text { membrane, maintain the attachment } \\
\text { of the nuclear membrane to nuclear } \\
\text { lamina during cell division }\end{array}$ & No & No \\
\hline PRR5L & Proline-rich protein 5-like & $\begin{array}{l}\text { Associate with mTORC2 complex } \\
\text { that regulate cellular processes } \\
\text { including survival and cytoskeleton } \\
\text { organization }\end{array}$ & $\mathrm{No}^{[35]}$ & No \\
\hline
\end{tabular}




\begin{tabular}{lllll}
\hline & & Bind and deliver cytosolic copper to & & \\
ATOX1 & Copper transport protein & $\begin{array}{l}\text { copper ATPase proteins, and be } \\
\text { important in cellular antioxidant } \\
\text { defense }\end{array}$ & Yes $^{[36,37]}$ & Yes $^{[38]}$ \\
\hline \multirow{2}{*}{ DTX3 } & Deltex E3 ubiquitin ligase 3 & $\begin{array}{l}\text { Function as an E3 ubiquitin ligase, } \\
\text { regulate Notch signaling }\end{array}$ & Yes $^{[39]}$ & No \\
\hline
\end{tabular}

2 